\title{
Inclusión social de las personas con discapacidad visual: Una revisión sistemática cualitativa
}

\author{
Ana Calle ${ }^{1}$, Amparito López ${ }^{1}$ y Maggie Campillay² \\ ${ }^{1}$ Departamento de Kinesiología de la Universidad de Atacama, Chile | Ana.calle@uda.cl; \\ amparolopeztoledo@gmail.com | https://orcid.org/0000-0002-8099-1486; \\ https://orcid.org/0000-0002-1178-9508 \\ 2 Departamento de Enfermería de la Universidad de Atacama, Chile | \\ Maggie.Campillay@uda.cl | https://orcid.org/0000-0002-4054-1595
}

Resumen: Objetivos: El objetivo fue identificar las relaciones interpersonales y las instancias de participación comunitaria que promueven el proceso de inclusión social de personas con discapacidad visual en la literatura existente. Métodos: Se realizó una revisión sistemática Integrativa con enfoque interpretativo meta-estudio de artículos publicados en las bases de datos, EBSCO, Web of Science (WOS), Biblioteca Virtual en Salud (BVS). Tras la búsqueda en las bases de datos, se seleccionó un total de 13 artículos primarios que cumplían con los criterios de inclusión. Resultados: el análisis de la información fue organizada en cuatro áreas categorías teóricas, acorde al objetivo de la investigación predefinido: (a) Discapacidad visual y relaciones interpersonales (b) Relaciones interpersonales en el proceso de inclusión social (c) Participación comunitaria en el proceso de inclusión social y (d) Barreras para la inclusión social. Conclusiones: se concluye que las relaciones interpersonales se dan principalmente con la familia. Existen barreras contextuales y actitudinales que han sido poco abordadas para eliminarlas. Las tecnologías de asistencia son una temática emergente con gran potencial para la inclusión. El uso de la revisión sistematizada con enfoque meta-estudio contribuyó a identificar áreas sustanciales para futuros estudios.

Palabras clave: Discapacidad Visual; Inclusión Social; Relaciones Interpersonales; Participación Comunitaria; Modelo Ecológico.

\section{Social Inclusion of Persons with Vision Disability: A Qualitative Systematic Review}

Abstract: Objectives: The objective was to identify interpersonal relationships and instances of community participation that promote the process of social inclusion of people with visual disabilities in the existing literature. Methods: A integrative systematic revision was carried out with an interpretative approach meta-studio of articles published in the databases, EBSCO, Web of Science (WOS), Virtual Health Library (VHL). After searching the databases, a total of 13 primary articles that met the inclusion criteria. Results: the information analysis was organized into four areas, theoretical categories, according to the predefined research objective: (a) Visual impairment and interpersonal relationships (b) Interpersonal relationships in the process of social inclusion (c) Community participation in the process of social inclusion and (d) Barriers to social inclusion. Conclusions: it is concluded that interpersonal relationships occur mainly with the family. There are contextual and attitudinal barriers that have been little addressed to eliminate them. Assistive technologies are an emerging topic with great potential for inclusion. The use of the systematic review with a meta-study approach helped to identify substantial areas for future studies.

Keywords: Community Involvement; Vision Impairment; Social Inclusion; Personal Relationships; Developmental Disabilities; Ecological Model.

\section{Introducción}

La revisión de la literatura permite una búsqueda detallada y selectiva de información acumulada sobre un fenómeno de interés. Esta metodología toma relevancia, cuando se trata de indagar en una temática poco estudiada, permitiendo a los autores situarse desde una perspectiva crítica (Guirao Goris, 2015). Se ha estudiado poco la influencia que tiene la discapacidad visual en la vida de las personas con esta condición, sus familias y/o contexto en el cual se desenvuelven (Fiorati \& Elui, 2015). 
La necesidad de conocer en profundidad la influencia de las relaciones interpersonales y participación de personas con discapacidad visual en la comunidad (Grilo \& Major, 2015), social, laboral entre otros, a través de una revisión, contribuiría a contar con una perspectiva integral del proceso de inclusión social, basada en la escasa evidencia científica que ha abordado el tema en cuestión hasta el momento (Perez et al., 2018).

Se ha establecido que adultos jóvenes con discapacidad visual dan gran valor a las actividades relacionadas con el trabajo y el estudio, las habilidades sociales y las relaciones sociales. Sin embargo, hay poca evidencia sobre el fenómeno de la participación de adultos jóvenes con discapacidad visual, su transición a la vida adulta y cómo está puede mejorar su calidad de vida (Elsman et al., 2017).

La discapacidad visual es una de las principales causas de limitación funcional en las personas, y una de las que presenta mayor prevalencia a nivel global (Stevens et al., 2013). La Organización Mundial de la Salud (OMS) considera que las deficiencias visuales provocan limitaciones en la realización de las tareas cotidianas, con consecuencias negativas para la calidad de vida de estas personas y sus familias (Organización Mundial de la salud, 2012).

En la actualidad, predomina todavía en la sociedad el paradigma médico, que proporciona barreras de diversa índole y limita la inclusión de personas con discapacidad visual, otorgándoles un rol pasivo en la comunidad (Santos, 2016). Al respecto, se han implementado estrategias en cada país para lograr una mayor inclusión social de las personas con discapacidad, pero estos esfuerzos van enfocados principalmente hacia personas con discapacidad física, excluyendo o postergando las de origen sensitivo, como la visual (Boudeguer \& Squella, 2010). Si bien ha mejorado la accesibilidad y se fomentado la participación, no se ha probado la eficacia de todas estas (Heppe et al.)

A nivel conceptual, existen numerosas definiciones de inclusión social, reportando usos intercambiables con integración social, redes sociales, participación comunitaria y capital social (Simplican et al., 2015). Esta variación entre las definiciones impide la prestación efectiva de servicios e intervenciones, y conduce a datos confusos e insuficientes para implementar acciones y estrategias efectivas comunes (Duggan \& Linehan, 2013; Simplican et al., 2015). Si bien el capital social es parte de la inclusión social, al proporcionar acceso a recursos externos y relaciones interpersonales con personas de identidad diversa no son conceptos sinónimos (Bassey et al., 2019). Por tanto, revisar la literatura contribuye a la identificación y discusión de los conceptos relevantes en la investigación sobre inclusión en personas con discapacidad.

Dada lo urgente de lograr una inclusión exitosa, se da cada vez mayor importancia a las relaciones interpersonales, el capital social y la participación comunitaria, como componentes claves declarados en la Convención de las Naciones Unidas sobre los Derechos de personas con discapacidad (ONU, 2006; Tobias \& Mukhopadhyay, 2017). Además de ser una cuestión de participación de derechos civiles y de igualdad de oportunidades (Simplican et al., 2015).

Estudios a nivel global, mencionan que las personas con discapacidad visual continúan experimentando discriminación y formas de exclusión social (Jessup et al., 2018; Tobias \& Mukhopadhyay, 2017). En este sentido, aportar con estudios que identifiquen las barreras actitudinales y ambientales que les afectan, y la forma de disminuirlas, contribuirían siempre a mayor igualdad, autonomía personal, y lograr mayor participación comunitaria (Phillips et al., 2019).Es de interés por tanto, para las propias personas con discapacidad visual no ser incluidos dentro de la sociedad, para esto es necesario conocer como se produce la inclusión social de este grupo en los más variados contextos.

Basado en el modelo ecológico que considera la inclusión social como la interacción entre dos dimensiones fundamentales de la vida: las relaciones interpersonales y la participación comunitaria (Simplican et al., 2015). 
A partir de ello, se plantea el principal objetivo de esta revisión sistematizada cualitativa de la literatura es identificar en la literatura existente las relaciones interpersonales y las instancias de participación comunitaria que promueven el proceso de inclusión social de las personas con discapacidad visual.

\section{Metodología}

Estudio es secundario y utilizó metodología de Revisión Sistemática Integrativa de métodos mixtos, para evaluar y sintetizar la evidencia para una comprensión completa del fenómeno analizado (Grant \& Booth, 2009; Guirao Goris, 2015), se realizó un meta estudio, basado en la evidencia de estudios cualitativos primarios y cuantitativos disponibles, y en la temática de relaciones interpersonales y participación comunitaria de las personas con discapacidad visual.

La revisión se realizó durante el periodo de Marzo al 9 de Mayo 2018, y consideró como referente metodológico la guía de revisión sistemática y síntesis cualitativas de Saini \& Shlonsky (Saini \& Shlonsky, 2012), quienes proponen un enfoque interpretativo de metaestudio, que permite realizar una pregunta predefinida que orienta la búsqueda y análisis de contenido de los artículos seleccionados. Para guiar el estudio y su discusión, se formuló la siguiente pregunta guía: ¿Cuáles son las relaciones interpersonales y las instancias de participación comunitaria que promueven el proceso de inclusión social de las personas con discapacidad visual? Esto incluye responder a los retos de revisión centrales, incluyendo estudios cualitativos y cuantitativos.

\subsection{Estrategia de Búsqueda}

La estrategia de búsqueda incluyo los trabajos publicados en un periodo comprendido en los últimos años, entre el 2013 y 2018, con relevancia frente a la pregunta de investigación. Se realizó la búsqueda en bases de datos electrónicos: EBSCO, Web of Science (WOS), BVS (Biblioteca Virtual en Salud), que indagarán en la inclusión social, las relaciones interpersonales y participación comunitaria como parte del proceso de inclusión social de las personas con discapacidad visual. Las palabras claves utilizadas fueron en español; discapacidad visual o inclusión social o relaciones interpersonales o participación comunitaria, así como sus correlativos en inglés; visual disability OR social inclusión OR Interpersonal relationships OR community participation. Los registros identificados en las búsquedas están realizados con gestor de referencias EndNote X8, donde se identificaron y eliminaron los duplicados.

\subsection{Criterios de Inclusión y Exclusión}

Criterios de Inclusión para el proceso de selección son los siguientes: El tipo de estudio Cualitativos, cuantitativos, mixtos; Primarios y secundarios. Tipo de indicadores: estudios que incluyan las palabras claves VISUAL DISABILITY, SOCIAL INCLUSION, INTERPERSONAL RELATIONSHIPS, COMMUNITY PARTICIPATION. Perfil: Población adulta con discapacidad visual. Criterio temporal: Publicaciones posterior al año 2013. Criterio lingüístico: Publicaciones en inglés, español y portugués. Criterio geográfico: Publicaciones a nivel global.

Los criterios de exclusión consideran estudios centrados en personas con múltiples patologías, estudios de caso único y estudios que se centren en una patología específica médica. 


\subsection{Análisis de Datos}

Se realizó estrategia de búsqueda y extracción de los datos en paralelo, a cargo de dos de las investigadoras principales realizando triangulación metodológica y de datos, con el fin de dar mayor confiabilidad a la revisión. El proceso de selección Fig.1., en primera ronda, se seleccionan los resultados a partir de la lectura de título y abstract, además de cumplir con los criterios de inclusión definidos. En segunda ronda, se realiza lectura exhaustiva de los artículos seleccionados en la etapa anterior, considerando que respondieran a la pregunta de investigación y aplicando los criterios de exclusión. En tercer ronda, se realizó el análisis crítico de los artículos seleccionados utilizando un formulario de extracción de datos tipo matriz Tabla 1, que incluye datos de información bibliográfica: autoría, año de publicación, título del artículo; tipo de estudio, primario o secundario; principal objetivo; criterios de calidad o rigor científico (para estudios primarios); caracterización de la muestra (para estudios primarios) (Lewin et al., 2015).

La interpretación se realizó considerando como método la técnica de análisis temáticocategórico, la creación de categorías teóricas que dieran respuesta a la pregunta de investigación, se utiliza como base el modelo ecológico de Simplican, 2015, quien considera a la inclusión social como la interacción entre dos dominios principales de la vida: las relaciones interpersonales y la participación comunitaria. Para ello, se utilizó la frecuencia de los verbatim solo para orientar la importancia dada en los artículos a las temáticas (Saini \& Shlonsky, 2012), ya que primó la interpretación de los investigadores.

Proceso de Selección

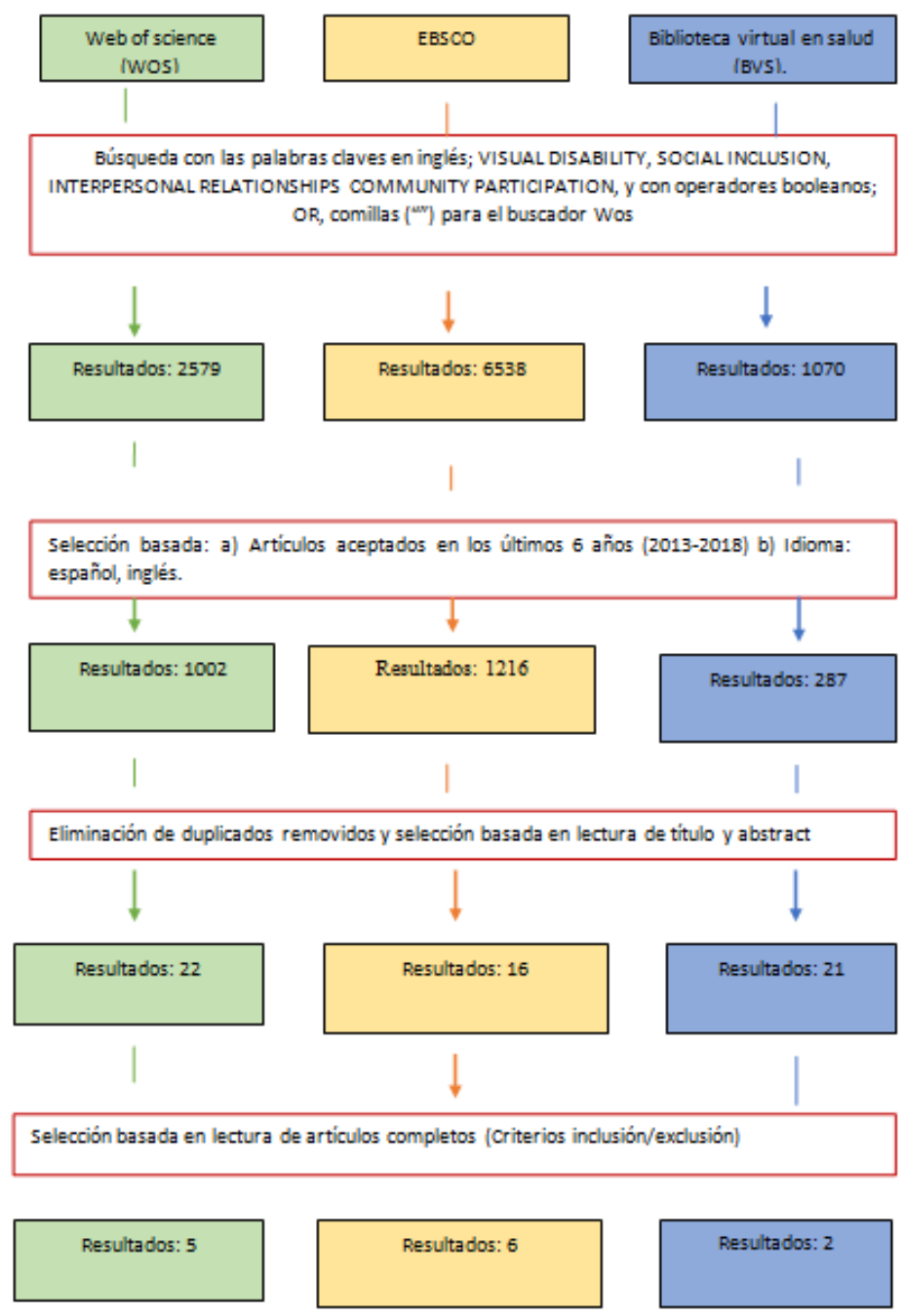

Fig. 1. Diagrama de flujo del proceso de selección. 


\section{Resultados}

Tras la búsqueda en las bases de datos analizadas, se seleccionó un total de 13 artículos primarios que cumplían con los criterios de inclusión y por la falta de artículos primarios se seleccionaron 5 artículos secundarios de revisiones que trataban la temática de estudio, de manera de contrastar los hallazgos. En la tabla 1 se puede ver las principales características de los estudios analizados en la revisión.

Tabla 1. Principales características de los estudios analizados en la revisión.

\begin{tabular}{|c|c|c|c|c|c|}
\hline Autor/es & Nombre del artículo & $\begin{array}{l}\text { Tipo de } \\
\text { estudio } \\
\text { (Primario- } \\
\text { Secundario) }\end{array}$ & Muestra & Propósito & $\begin{array}{c}\text { Calidad } \\
\text { Criterios de rigor }\end{array}$ \\
\hline $\begin{array}{ll}\text { Opoku, } & \text { M., } \\
\text { Alupo, } & \text { B., } \\
\text { Gyamfi, } & \text { N., } \\
\text { Odame, } & \text { L., } \\
\text { Mprah, } & \text { W., } \\
\text { Torgbenu, } & \text { E., } \\
\text { \& Badu, } & \text { E. } \\
\text { (2018). } & \end{array}$ & $\begin{array}{l}\text { The Family and Disability } \\
\text { in Ghana: Highlighting } \\
\text { Gaps in Achieving Social } \\
\text { Inclusion. }\end{array}$ & Primario & $\begin{array}{l}\text { De } 48 \text { participantes una vez } \\
\text { que se alcanza la saturación } \\
\text { de datos. } \\
4 \text { capitales de distrito } \\
\text { Todos ellos miembros } \\
\text { Disabled } \\
\text { Peoples' Organizaciones }\end{array}$ & $\begin{array}{l}\text { Documentar las } \\
\text { perspectivas de las } \\
\text { personas } \\
\text { con discapacidad en el } \\
\text { impacto del apoyo } \\
\text { familiar - o su ausencia } \\
\text { - en su vida adulta en } \\
\text { Ghana. }\end{array}$ & $\begin{array}{l}\text { Auditabilidad, los } \\
\text { autores siguieron } \\
\text { los pasos a través } \\
\text { de lectura, datos, } \\
\text { organización y } \\
\text { codificación. }\end{array}$ \\
\hline $\begin{array}{l}\text { Nakade, A., } \\
\text { Rohatgi, J., } \\
\text { Bhatia, M. S., } \\
\text { \& Dhaliwal, } \\
\text { U. (2017). }\end{array}$ & $\begin{array}{l}\text { Adjustment to acquired } \\
\text { vision loss in adults } \\
\text { presenting for visual } \\
\text { disability certification }\end{array}$ & Primario & $\begin{array}{l}\text { De } 30 \text { personas reclutadas, } \\
24 \text { eran hombres ( } 80 \%) ; 24 \\
\text { vivian en areas urbanas } \\
(80 \%) ; 9 \text { fueron empleados } \\
(30 \%) ; \text { y } 14(46.6 \%) \text { habian } \\
\text { estudiado <Clase } 3\end{array}$ & $\begin{array}{l}\text { Evaluar el ajuste a la } \\
\text { pérdida de visión } \\
\text { adquirida en adultos. } \\
\text { Comprender los factores } \\
\text { contribuyentes puede } \\
\text { ayudar en la } \\
\text { rehabilitación efectiva. }\end{array}$ & $\begin{array}{l}\text { Validez, el efecto } \\
\text { de la edad en el } \\
\text { ajuste se calculó } \\
\text { utilizando } \\
\text { correlación la } \\
\text { Pearson. La } \\
\text { correlación entre el } \\
\text { puntaje de ajuste } \\
\text { total, el puntaje de } \\
\text { depresión, el } \\
\text { puntaje de } \\
\text { personalidad y el } \\
\text { apoyo social y los } \\
\text { puntajes de estrés } \\
\text { se calcularon } \\
\text { usando la } \\
\text { correlación de } \\
\text { Spearman. Los }\end{array}$ \\
\hline & & & & & $\begin{array}{l}\text { resultados se } \\
\text { consideraron } \\
\text { estadisticamente } \\
\text { significativos } \\
\text { a } P<0.05 \text {. }\end{array}$ \\
\hline $\begin{array}{l}\text { Mendez- } \\
\text { Ulrich, J. L., } \\
\text { Prats-Basset, } \\
\text { L., Yague, F., } \\
\text { \& Sanz, A. } \\
(2016) .\end{array}$ & $\begin{array}{lrr}\text { Perceived } & \text { control, } & \text { coping } \\
\text { and } & \text { psychological } \\
\text { adjustment } & \text { to } & \text { visual } \\
\text { disability. } & & \end{array}$ & Primario & $\begin{array}{l}\text { De } 35 \text { personas ( } 15 \text { hombres } \\
\text { y } 20 \text { mujeres), miembros de } \\
\text { la Asociación Discapacidad } \\
\text { Visual Catalunya. En este } \\
\text { estudio se empleó un } \\
\text { muestreo por conveniencia. } \\
\text { La media de edad de los } \\
\text { participantes fue de } 56,2 \\
\text { años que accedieron } \\
\text { voluntariamente a participar } \\
\text { en el estudio. }\end{array}$ & $\begin{array}{l}\text { Se emplé un diseño } \\
\text { transversal ex post facto } \\
\text { descriptivo y } \\
\text { correlacional entre la } \\
\text { competencia percibida y } \\
\text { el ajuste psicológico a la } \\
\text { discapacidad visual. } \\
\text { Además, se evaluó el } \\
\text { posible efecto de } \\
\text { diversas posibles } \\
\text { covariables en la } \\
\text { relación entre la } \\
\text { competencia percibida y } \\
\text { el ajuste psicológico, } \\
\text { como el tipo de } \\
\text { discapacidad. }\end{array}$ & $\begin{array}{l}\text { Fiabilidad, } \\
\text { fiabilidad de las } \\
\text { pruebas } \\
\text { psicométricas } \\
\text { empleadas en su } \\
\text { adaptación al } \\
\text { formato de } \\
\text { administración } \\
\text { oral, que igualan } \\
\text { prácticamente los } \\
\text { valores obtenidos } \\
\text { en su modalidad } \\
\text { autoaplicada con } \\
\text { muestra normativa. }\end{array}$ \\
\hline
\end{tabular}




\begin{tabular}{|c|c|c|c|c|c|}
\hline $\begin{array}{l}\text { Andrade, A., } \\
\text { Silva, I., \& } \\
\text { Veloso, A. } \\
\text { (2017). }\end{array}$ & $\begin{array}{l}\text { Integração profissional de } \\
\text { pessoas com deficiência } \\
\text { visual: Das práticas } \\
\text { organizacionais às atitudes } \\
\text { individuais }\end{array}$ & Primario & $\begin{array}{l}\text { Se entrevistaron a } 13 \\
\text { personas, } 8 \text { trabajadores y } 5 \\
\text { jefes de los primeros. Todos } \\
\text { los trabajadores eran } \\
\text { ciegos. De las } 5 \text { jefaturas, } 2 \\
\text { también eran ciegas. }\end{array}$ & $\begin{array}{l}\text { Contribuir al } \\
\text { conocimiento sobre la } \\
\text { integración, así como } \\
\text { reflexionar sobre las } \\
\text { políticas y prácticas que } \\
\text { fomentan dicha } \\
\text { integración. }\end{array}$ & $\begin{array}{l}\text { Auditabilidad, } \\
\text { metodologia de } \\
\text { análisis cualitativo, } \\
\text { datos, organización } \\
\text { y codificación. }\end{array}$ \\
\hline $\begin{array}{l}\text { Raymond, E., } \\
\text { Grenier, A., \& } \\
\text { Hanley, J. } \\
\text { (2014). }\end{array}$ & $\begin{array}{l}\text { Community Participation of } \\
\text { Older Adults with } \\
\text { Disabilities. }\end{array}$ & Primario & $\begin{array}{l}\text { De } 12 \text { participantes } \\
\text { proyecto basado en la } \\
\text { comunidad en la forma } \\
\text { de una fotonovela. }\end{array}$ & \begin{tabular}{|l} 
El estudio refleja cómo \\
es el envejecimiento de \\
las personas con \\
discapacidades e \\
interpretar la \\
experiencia de \\
participación social en \\
el contexto de la agenda \\
de política participativa.
\end{tabular} & $\begin{array}{lr}\text { Se utilizaron } & \text { tres } \\
\text { estrategias } & \text { para } \\
\text { desarrollar } & \text { la } \\
\text { Confiabilidad } & \text { a } \\
\text { través } & \text { de } \\
\text { participación } & \\
\text { prolongada con los } \\
\text { participantes } \\
\text { triangulación. }\end{array}$ \\
\hline $\begin{array}{l}\text { Giesen, J. M., } \\
\text { \& Cavenaugh, } \\
\text { B. S. (2013). }\end{array}$ & $\begin{array}{l}\text { Disability Insurance } \\
\text { Beneficiaries with Visual } \\
\text { Impairments in Vocational } \\
\text { Rehabilitation: Socio- } \\
\text { demographic Influences on } \\
\text { Employment. }\end{array}$ & Primario & $\begin{array}{l}\text { De } 4.478 \text { personas del } \\
\text { Seguro de Discapacidad de } \\
\text { Seguro Social (SSDI) }\end{array}$ & 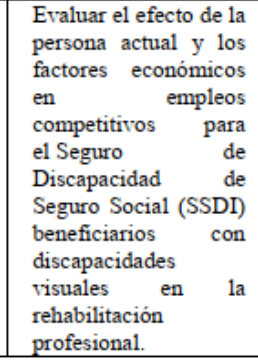 & 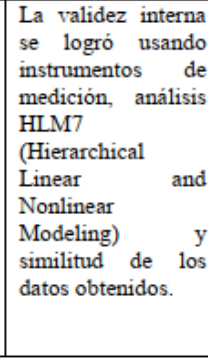 \\
\hline $\begin{array}{l}\text { Fiorati, R. C., } \\
\text { \& Elui, V. M. } \\
\text { C. (2015) }\end{array}$ & $\begin{array}{l}\text { Los determinantes sociales } \\
\text { de la salud, desigualdad e } \\
\text { inclusión social entre las } \\
\text { personas con discapacidad. }\end{array}$ & Primario & $\begin{array}{l}\text { De } 10 \text { personas con } \\
\text { discapacidad congénita o } \\
\text { adquirida que viven en la } \\
\text { región cubierta por un centro } \\
\text { de salud familiar. }\end{array}$ & 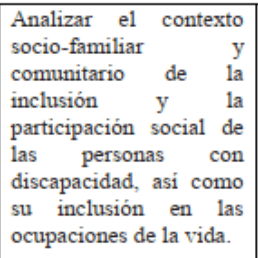 & $\begin{array}{l}\text { Confiabilidad, } \\
\text { ordenación de } \\
\text { datos y su continuo } \\
\text { análisis. }\end{array}$ \\
\hline $\begin{array}{l}\text { Cazini, J., \& } \\
\text { Frasson, A. C. } \\
(2013\end{array}$ & $\begin{array}{l}\text { Proyecto Voces: } \\
\text { Innovaciones tecnológicas } \\
\text { en la Inclusión Social de las } \\
\text { Personas con Discapacidad } \\
\text { Visual. }\end{array}$ & Primario & $\begin{array}{l}20 \text { estudiantes en edades de } \\
18 \text { a } 54 \text { años. }\end{array}$ & $\begin{array}{lr}\text { Hacer posible } & \text { la } \\
\text { accesibilidad } & \text { a } \\
\text { la información } & \\
\text { mediante el uso de } & \text { de } \\
\text { programas } & \text { de } \\
\text { computadora } & \text { que } \\
\text { pueden apoyar las } \\
\text { actividades educativas, } \\
\text { favoreciendo } \\
\begin{array}{lr}\text { capacitación la } \\
\text { para actividades }\end{array} \\
\text { profesionales. }\end{array}$ & $\begin{array}{lr}\text { Proyecto } & \text { Voces } \\
\text { informa que este } \\
\text { articulo es } & \text { un } \\
\text { ejemplo } & \text { de } \\
\text { aplicabilidad. } & \end{array}$ \\
\hline $\begin{array}{l}\text { Grilo, I., \& } \\
\text { Major, S. } \\
(2015)\end{array}$ & $\begin{array}{l}\text { Calidad de vida de la } \\
\text { familia, la satisfacción con } \\
\text { la vida y el apoyo social } \\
\text { percibido en Discapacidad } \\
\text { Visual }\end{array}$ & Primario & $\begin{array}{l}\text { Se necesitaron dos muestras } \\
\text { de sujetos con edad igual o } \\
\text { superior a } 18 \text { años: los } \\
\text { sujetos adultos con } \\
\text { diagnóstico de discapacidad } \\
\text { visual moderada el grupo } \\
\text { total (DV) y sujetos }\end{array}$ & 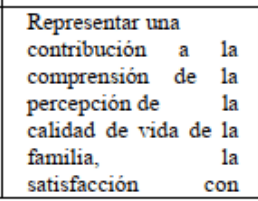 & $\begin{array}{l}\text { Validez interna, se } \\
\text { usó el coeficiente } \\
\text { alfa de Cronbach. }\end{array}$ \\
\hline
\end{tabular}


Vol. 8 | Investigación Cualitativa en Salud: Avances y Desafíos

\begin{tabular}{|c|c|c|c|c|c|}
\hline & & & $\begin{array}{l}\text { adultos normovisual (grupo } \\
\text { de comparación) }\end{array}$ & $\begin{array}{l}\text { la vida y el apoyo } \\
\text { social percibido entre } \\
\text { personas con } \\
\text { discapacidad visual y } \\
\text { sujetos normovisuales }\end{array}$ & \\
\hline $\begin{array}{lr}\text { Mazer, } & \text { B., } \\
\text { Kairy, } & \text { D., } \\
\text { Guindon, } & \text { A., } \\
\text { Girard, } & \text { M., } \\
\text { Swaine, } & \text { B., } \\
\text { Kehayia, } & \text { E., \& } \\
\text { Labbé, } & \text { D. } \\
\text { (2015). } & \end{array}$ & $\begin{array}{l}\text { Rehabilitation living } \\
\text { lab in the mall } \\
\text { community of } \\
\text { practice: learning } \\
\text { together to improve } \\
\text { rehabilitation, } \\
\text { participation and } \\
\text { social inclusion for } \\
\text { people living with } \\
\text { disabilities }\end{array}$ & Primario & $\begin{array}{l}43 \text { participantes en la } \\
\text { recopilación de datos en } \\
\text { linea y } 28 \text { participantes } \\
\text { tomaron parte en el } \\
\text { cuestionario y entrevista }\end{array}$ & $\begin{array}{lr}\text { Aumentar } & \text { la } \\
\text { participación social de } \\
\text { las personas con } \\
\text { discapacidad. Exponer } \\
\text { la necesidad de incluir a } \\
\text { partes interesadas en las } \\
\text { fases iniciales del } \\
\text { proceso } & \text { de } \\
\text { investigación } & \text { para } \\
\text { desarrollar proyectos } & \\
\text { pertinentes a r sus } \\
\text { necesidades. }\end{array}$ & $\begin{array}{lr}\text { Credibilidad } & \text { a } \\
\text { través } & \text { de } \\
\text { triangulación } & \end{array}$ \\
\hline $\begin{array}{l}\text { Peres, R, } \\
\text { Espirito-Santo, } \\
\text { G., Espirito, } \\
\text { F., Ferreira, } \\
\text { N., \& Assis, } \\
\text { M. (2015). }\end{array}$ & $\begin{array}{l}\text { Insatisfaçào com a } \\
\text { imagem corporal } \\
\text { entre pessoas com } \\
\text { deficiência visual }\end{array}$ & Primario & $\begin{array}{l}45 \text { sujetos en la encuesta } \\
\text { con discapacidades } \\
\text { patronos del Instes } \\
\text { Benjamin Constant. }\end{array}$ & $\begin{array}{l}\text { Comprobar una } \\
\text { insatisfacción con una } \\
\text { imagen corporal en } \\
\text { sujetos con ceguera, } \\
\text { congénitas y adquiridas. }\end{array}$ & $\begin{array}{l}\text { Validez interna } \\
\text { debido a similitud } \\
\text { entre los datos } \\
\text { obtenidos y la } \\
\text { realidad. }\end{array}$ \\
\hline 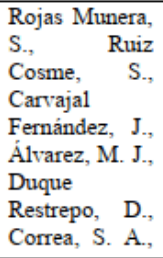 & $\begin{array}{l}\text { Caracterización de una } \\
\text { población con discapacidad } \\
\text { visual (baja visión y } \\
\text { ceguera) atendida en dos } \\
\text { Instituciones Prestadoras de } \\
\text { Salud de Medellin }\end{array}$ & Primario & 107 historias clinicas & $\begin{array}{l}\text { Caracterizar, en los } \\
\text { ámbitos clinico y } \\
\text { sociodemográfico, una } \\
\text { población de pacientes } \\
\text { con discapacidad visual } \\
\text { atendidos en dos } \\
\text { instituciones de salud de } \\
\text { la ciudad de Medellin. }\end{array}$ & $\begin{array}{l}\text { Validez interna, } \\
\text { debido a la } \\
\text { similitud de datos } \\
\text { obtenidos con la } \\
\text { realidad. }\end{array}$ \\
\hline \multicolumn{6}{|l|}{$\begin{array}{l}\text { Ramirez } \\
\text { Rodriguez, C., } \\
\text { Pineda } \\
\text { Jiménez, L., } \\
\text { Restrepo } \\
\text { Arismendy, S., } \\
\text { Castrillón } \\
\text { Valencia, L. } \\
\text { C., Arango } \\
\text { Valencia, S., } \\
\text { Portela, M. C., } \\
\text { Fernández } \\
\text { Zurita, M., } \\
\text { Astudillo } \\
\text { Valverde, E., } \\
\text { Galarcio, J. F., } \\
\text { \& Suárez- } \\
\text { Escudero, J. C. } \\
\text { (2015). }\end{array}$} \\
\hline $\begin{array}{l}\text { Perez, F. J., } \\
\text { Romeo, M., \& } \\
\text { Yepes-Baldó, } \\
\text { M. (2018). }\end{array}$ & $\begin{array}{l}\text { The corporate social } \\
\text { responsibility policies for } \\
\text { the inclusion of people with } \\
\text { disabilities as predictors of } \\
\text { employees identification, } \\
\text { commitment and } \\
\text { absenteeism }\end{array}$ & Primario & $\begin{array}{c}104 \\
\text { Empleados RSC }\end{array}$ & $\begin{array}{l}\text { E1 efecto en los } \\
\text { empleados de las } \\
\text { politicas de } \\
\text { Responsabilidad Social } \\
\text { Corporativa (RSC) } \\
\text { orientadas a la inclusión } \\
\text { de las personas con } \\
\text { discapacidades. }\end{array}$ & $\begin{array}{l}\text { Su consistencia } \\
\text { interna y validez } \\
\text { de constructo se } \\
\text { realizo mediante } \\
\text { alfa de Cronbach y } \\
\text { análisis factorial } \\
\text { exploratorio. }\end{array}$ \\
\hline
\end{tabular}




\begin{tabular}{|c|c|c|c|}
\hline Autor/es & Título & Tipo de Estudio & Objetivo \\
\hline Santos, W. (2016). & $\begin{array}{lr}\text { Deficiência } & \text { como } \\
\text { restrição } & \text { de } \\
\text { participação } & \text { social: } \\
\text { desafios para avaliação } \\
\text { a partir da Lei Brasileira } \\
\text { de Inclusão. }\end{array}$ & Secundario & $\begin{array}{l}\text { Analizar los principales avances y desafios } \\
\text { para la comprensión y valoración de la } \\
\text { discapacidad como una limitación para la } \\
\text { participación social. }\end{array}$ \\
\hline $\begin{array}{l}\text { Alarcón, C., \& Vizcarra, M. } \\
\text { (2016). }\end{array}$ & $\begin{array}{l}\text { Personas en situación } \\
\text { de discapacidad } \\
\text { visual en relación a } \\
\text { las barreras y } \\
\text { estrategias que } \\
\text { afectan r las } \\
\text { actividades de la vida } \\
\text { diaria intrumentales. }\end{array}$ & Secundario & $\begin{array}{l}\text { Conocer la información disponible sobre el } \\
\text { desempeño en las actividades de la vida } \\
\text { diaria instrumentales (AVDI) de las } \\
\text { personas en situación de discapacidad } \\
\text { visual en relación a las barreras que } \\
\text { presentan para su ejecución. }\end{array}$ \\
\hline $\begin{array}{l}\text { Oliveira, C. M., \& Nunes, C. } \\
\text { H. S. d. S. (2015). }\end{array}$ & $\begin{array}{l}\text { Instrumentos para } \\
\text { Avaliação Psicológica de } \\
\text { Pessoas com Deficiência } \\
\text { Visual: Tecnologias para } \\
\text { Desenvolvimento e } \\
\text { Adaptação }\end{array}$ & Secundario & $\begin{array}{l}\text { Reconocer la importancia de las } \\
\text { adaptaciones, discutir la aplicación de los } \\
\text { recursos tecnológicos como una forma de } \\
\text { habilitar la accesibilidad y presentar la } \\
\text { propuesta de diseño universal como } \\
\text { potencializadora en materia de } \\
\text { discapacidad visual. }\end{array}$ \\
\hline $\begin{array}{lr}\text { World, } & \text { Health, } \\
\text { Organization. (2013). }\end{array}$ & $\begin{array}{l}\text { Sixty-sixth World Health } \\
\text { Assembly A66/11. Action } \\
\text { plan project to prevent } \\
\text { avoidable blindness and } \\
\text { vision impairment 2014- } \\
2019 \text {. }\end{array}$ & Secundario & $\begin{array}{l}\text { Reducir la discapacidad visual evitable } \\
\text { como problema de salud pública mundial } \\
\text { y garantizar el acceso a los servicios de } \\
\text { rehabilitación para los discapacitados } \\
\text { visuales. }\end{array}$ \\
\hline $\begin{array}{l}\text { Deliyore-Vega, M. } \quad \text { d. } \quad \text { R. } \\
\text { (2018). }\end{array}$ & $\begin{array}{l}\text { Comunicación alternativa, } \\
\text { herramienta para la } \\
\text { inclusión social de las } \\
\text { personas en condición de } \\
\text { discapacidad }\end{array}$ & Secundario & $\begin{array}{l}\text { Determinar la relación que se da entre los } \\
\text { supuestos teóricos sobre procesos } \\
\text { inclusivos y su vinculación con la } \\
\text { comunicación alternativa }\end{array}$ \\
\hline
\end{tabular}

La descripción de los artículos fue organizada en cuatro categorías teóricas, siguiendo el objetivo de investigación: (a) Discapacidad visual y relaciones interpersonales (b) relaciones interpersonales en el proceso de inclusión social (c) Participación comunitaria en el proceso de inclusión social de personas con discapacidad visual y (d) Barreras para la inclusión social en las personas con discapacidad.

\subsection{Discapacidad Visual y las Relaciones Interpersonales}

Los artículos exponen que la discapacidad visual es un tema amplio, de interés epidemiológico, clínico, asistencial y socio cultural, con retos en cuanto a promociónprevención, tratamiento y rehabilitación que exige una comprensión biopsicosocial (Rojas Munera et al., 2015). De acuerdo con el grado de limitación de visión que presentan se puede distinguir entre personas ciegas, donde existe una pérdida completa del sentido de la vista, y personas con disminución visual, donde hay una pérdida parcial del sentido de la vista (Andrade et al., 2017). Se señala que las afectaciones no sólo son para el individuo, sino para la familia, y la incapacidad de ampliar su red de contactos interpersonales disminuye las redes de apoyo disponibles (Grilo \& Major, 2015).

Peres indica que es importante diferenciar entre una persona que nace con una limitación visual total o parcial, y una persona que adquiere la limitación en algún momento de su vida, esto condiciona formas distintas en la manera de relacionarse. Las personas que nacieron ciegas no tuvieron acceso a la información visual y por lo tanto desde temprana edad logran desarrollar el tacto para conocer, comunicarse y mantener relaciones interpersonales que le permite actuar de manera más autónoma. 
Respecto a las personas que presentaron una discapacidad visual posterior, Raymond (27) menciona que presentan mayor dificultad a la hora de mantener relaciones interpersonales, debido a que requieren de adaptaciones y mayores ajustes del entorno para socializar, tales como cambiar la ubicación de una reunión, tener accesos en dependencias de primer piso etc., estas adaptaciones frecuentemente tardan mucho tiempo en ser atendidas y no son ajustadas razonablemente, por lo tanto, se trasforma en una experiencia de vida perturbadora.

\subsection{Relaciones Interpersonales en el Proceso de Inclusión Social}

Las relaciones interpersonales abarcan un conjunto de recursos alcanzados en el contexto de las relaciones sociales, donde el sujeto accede a situaciones que le generan estrés y ansiedad. En este contexto, la familia y amigos son quienes se mencionan con mayor presencia en la vida social de las personas ciegas, entregando contención y empatía (Grilo \& Major, 2015; Opoku et al., 2018). La familia es por tanto, quien ofrece un apoyo incondicional, cuidado y crea una conexión específica de contacto interpersonal (Nakade et al., 2017). A partir de ello, la persona con discapacidad genera lazos de confianza, sin el temor a ser dañado en su dignidad, abriendo las posibilidades para que sirva como un medio de inclusión social (Peres et al., 2015).

En la revisión aparecen distintos modelos de familia, algunos muy sobreprotectores que van a tener una influencia negativa en el comportamiento social del individuo, manteniéndolos en un estado de pasividad y resguardo, mientras que otras familias permiten y fomentan la independencia de las personas con discapacidad visual proporcionando un mayor desarrollo personal y mejor autoestima que le permitirá relacionarse con más confianza y al mismo tiempo ser socialmente más eficaz (Meadan et al., 2010). Cuando existen riesgos en las familias, se sugiere asesoramiento especializado para manejar mejor las emociones, y capacitar a los miembros para responder a las necesidades emocionales de las personas con discapacidad visual, esto favorecería las relaciones a futuro (Nakade et al., 2017). Algunas familias que han renegado su responsabilidad de apoyar a sus familiares con discapacidad les privan de desarrollar sus potencialidades y de acceder a oportunidades de actividades productivas que puedan mejorar su condición de vida. La importancia de este elemento, es que las personas ciegas que no cuentan con apoyo familiar, pueden llegar al abandono, y dedicarse a labores como la mendicidad (Opoku et al., 2018), lo que daña su dignidad como persona.

Por tanto, la familia desempeñaría un papel significativo en garantizar el desarrollo de la personalidad, educación, participación y la utilización de los servicios públicos que necesitan las personas ciegas para su vida en autonomía (Opoku et al., 2018).

\subsection{Participación Comunitaria en el Proceso de Inclusión Social de las Personas con Discapacidad Visual}

La inclusión supone la participación de todas las personas, en igualdad de oportunidades, por lo tanto, se deben realizar en las primeras etapas de la vida, los ajustes que sean necesarios para asegurar la eliminación de barreras excluyentes. Por lo que se debe ofrecer alternativas y estrategias destinadas a promover la participación plena(Deliyore-Vega, 2018; Raymond et al., 2014; World et al., 2013).

La participación se da de acuerdo al ciclo vital del ser humano permitiéndole diversidad de experiencias e interacciones, con distintos niveles de satisfacción personal, se incluye como participación; descanso, juego, ocio, tiempo libre, trabajo (Alarcón \& Vizcarra, 2016). La principal forma de empoderar a las personas con discapacidad a tomar el control de su vida es cuando reciben apoyo para tener acceso a la educación, y así poder desenvolverse plenamente en el mercado laboral (Barton, 2009), ya sea con trabajo remunerado o no remunerado, como el voluntariado, que son formas activas de participación. 
También se mencionan diferencias en el aspecto laboral y profesional entre hombres y mujeres, siendo el sexo femenino el menos favorecido presentando una disminución en las oportunidades de empleo competitivo (Giesen \& Cavenaugh, 2013).

Estudio que consideraron los empleadores refieren que no contratan personas con discapacidad por temor a que puedan lesionarse, por considerar que no tienen capacidad para realizar el trabajo o, por temor a reacciones discriminatorias por parte de colegas y/o clientes (Andrade et al., 2017). Para las personas ciegas obtener un empleo afecta la dimensión psicológica y social, esto afectará la autonomía e integración plena en la sociedad (Andrade et al., 2017; Giesen \& Cavenaugh, 2013).

Se menciona mayor satisfacción entre el empleo y actividades de ocio, y menos satisfacción entre el empleo y las relaciones familiares. Esto podría deberse a que las personas ciegas, tal vez puedan sufrir diferentes dificultades a la hora de constituir su propia familia y en general en la participación familiar por falta de empleo (Mendez-Ulrich et al., 2016).

\subsection{Las Barreras que Impiden la Inclusión Social en las Personas con Discapacidad}

La sociedad presenta barreras de diversas índoles para las personas con discapacidad. Sin embargo, para las personas ciegas éstas barreras pueden presentarse más frecuentemente que en otras discapacidades, y tener un mayor impacto en el desarrollo de sus vidas (Alarcón \& Vizcarra, 2016; Mendez-Ulrich et al., 2016; Nakade et al., 2017; Raymond et al., 2014). Las barreras estructurales y de organización limitan su participación, haciéndolos sujetos pasivos en su naturaleza y amenazando su derecho a elegir y participar (Raymond et al., 2014). Esto causa falta de accesibilidad, al limitar la realización de las actividades de forma autónoma (Meadan et al., 2010).

Otros aspectos que dan cuenta de exclusión en la sociedad, es la falta de entrega de información técnica y acceso a tecnologías asistidas para facilitar la inclusión. La importancia de éstas, es que contribuyen con instrumentos y herramientas que permiten aminorar los obstáculos o barreras existentes, facilitando el acceso a personas con discapacidad visual (Mazer et al., 2015; Meadan et al., 2010). La implementación de herramientas como el sistema Braille, grabadora, programas de computación, audios entre otros, deben ser usados a diario para mejorar la comunicación (Alarcón \& Vizcarra, 2016; Cazini \& Frasson, 2013; Mazer et al., 2015). De esta forma, las capacidades y aptitudes se aproximan a las de un normovisual, asumiendo un papel activo tanto a nivel social como profesional (Oliveira \& Nunes, 2015).

Las barreras actitudinales tienen una gran influencia en el desarrollo personal (Alarcón \& Vizcarra, 2016), y se asocian a estigmas, discriminación y mal trato que son consideradas más nocivas que las barreras físicas (Grilo \& Major, 2015; Opoku et al., 2018).

\section{Conclusión}

Esta revisión contribuye a comprender como se da el proceso de inclusión social en las personas con discapacidad visual a partir de la literatura existente. Se evidencia que existen grandes brechas en el abordaje de esta población, y en las distintas áreas que promueven la inclusión. De los ámbitos abordados, las relaciones interpersonales se dan principalmente con la familia en un contexto más cercano a la persona con discapacidad visual, y en menor medida con amistades. La familia proporciona un importante apoyo emocional, y es el nexo para que se presente la participación comunitaria.

La participación representa un cambio positivo en la percepción de la vida de la persona con discapacidad, ya que accede a una mayor interacción con el medio y con posibilidades reales de inserción social y laboral, que le permiten ejercer sus derechos con autonomía. 
Se puede también concluir, que parte de las limitaciones que se presenta son producto de los atisbos del modelo médico que aún se mantiene imperante y que menoscaban las posibilidades reales de inclusión social.

Por tanto, la revisión sistematizada desde el enfoque de meta-estudio permitió integrar la evidencia disponible, contestar la pregunta de investigación basada en temas predefinidos, y orientar la búsqueda de datos. Como resultado, es posible identificar áreas sustantivas que pueden guiar investigaciones futuras, y lograr una interpretación con una mirada propia de los investigadores.

\section{Referencias}

Alarcón, C., \& Vizcarra, M. (2016). PERSONAS EN SITUACIÓN DE DISCAPACIDAD VISUAL EN RELACIÓN A LAS BARRERAS Y ESTRATEGIAS QUE AFECTAN LAS ACTIVIDADES DE LA VIDA DIARIA INSTRUMENTALES. Revista Chilena de Terapia Ocupacional, 16, 153. https://doi.org/10.5354/0719-5346.2016.44759

Andrade, A., Silva, I., \& Veloso, A. (2017). Integração profissional de pessoas com deficiência visual: Das práticas organizacionais às atitudes individuais. Revista Psicologia, Organizações e Trabalho, 17, 80-88. https://doi.org/10.17652/rpot/2017.2.12687

Barton, L. (2009). Studies on disability and the quest for inclusivity: Some observations [Article]. Revista De Educacion(349), 137-152. <Go to ISI>://WOS:000279256800007

Bassey, E., Ellison, C., \& Walker, R. (2019). Social capital, social relationships and adults with acquired visual impairment: a Nigerian perspective [Article]. Disability and Rehabilitation, 41(10), 1169-1176. https://doi.org/10.1080/09638288.2017.1423401

Boudeguer, \& Squella, P. (2010). Manual de Accesibilidad Universal. http:// www.ciudadaccesible.cl/wp-content/uploads/2012/06/manual_accesibilidad_universal1.pdf

Cazini, J., \& Frasson, A. C. (2013). Voices Project: Technological Innovations in Social Inclusion of People with Visual Impairment. Journal of technology management \& innovation, 8, 13-13.

Deliyore-Vega, M. d. R. (2018). Comunicación alternativa, herramienta para la inclusión social de las personas en condición de discapacidad. Revista Electrónica Educare, 22, 271-286.

Duggan, C., \& Linehan, C. (2013). The role of 'natural supports' in promoting independent living for people with disabilities; a review of existing literature [https://doi.org/10.1111/bld.12040]. British Journal of Learning Disabilities, 41(3), 199-207. https://doi.org/https://doi.org/10.1111/bld.12040

Elsman, E. B. M., van Rens, G., \& van Nispen, R. M. A. (2017). Impact of visual impairment on the lives of young adults in the Netherlands: a concept-mapping approach [Article]. Disability and Rehabilitation, 39(26), 2607-2618. https://doi.org/10.1080/09638288.2016.1236408

Fiorati, R. C., \& Elui, V. M. C. (2015). Social determinants of health, inequality and social inclusion among people with disabilities [Article]. Revista Latino-Americana De Enfermagem, 23(2), 329-336. https://doi.org/10.1590/0104-1169.0187.2559

Giesen, J. M., \& Cavenaugh, B. S. (2013). Disability Insurance Beneficiaries with Visual Impairments in Vocational Rehabilitation: Socio-demographic Influences on Employment. Journal of Visual Impairment \& Blindness, 107(6), 453-467. https://doi.org/10.1177/0145482X1310700607

Grant, M. J., \& Booth, A. (2009). A typology of reviews: an analysis of 14 review types and associated methodologies. Health Info Libr J, 26(2), 91-108. https://doi.org/10.1111/j.14711842.2009.00848.x

Grilo, I., \& Major, S. (2015). Qualidade de Vida Familiar, Satisfação com a Vida e Apoio Social Percebido Na Deficiência Visual. Temas em Psicologia, 23, 327-339. https://doi.org/10.9788/TP2015.2-07

Guirao Goris, S. J. A. (2015). Utilidad y tipos de revisión de literatura. Ene, 9, 0-0. 
Heppe, E. C. M., Willemen, A. M., Kef, S., \& Schuengel, C. Improving social participation of adolescents with a visual impairment with community-based mentoring: results from a randomized controlled trial [Article; Early Access]. Disability and Rehabilitation, 1-12. https://doi.org/10.1080/09638288.2019.1589587

Jessup, G. M., Bundy, A. C., Hancock, N., \& Broom, A. (2018). Being noticed for the way you are: Social inclusion and high school students with vision impairment [Article]. British Journal of Visual Impairment, 36(1), 90-103. https://doi.org/10.1177/0264619616686396

Lewin, S., Glenton, C., Munthe-Kaas, H., Carlsen, B., Colvin, C. J., Gülmezoglu, M., Noyes, J., Booth, A., Garside, R., \& Rashidian, A. (2015). Using Qualitative Evidence in Decision Making for Health and Social Interventions: An Approach to Assess Confidence in Findings from Qualitative Evidence Syntheses (GRADE-CERQual). PLOS Medicine, 12(10), e1001895. https://doi.org/10.1371/journal.pmed.1001895

Mazer, B., Kairy, D., Guindon, A., Girard, M., Swaine, B., Kehayia, E., \& Labbé, D. (2015). Rehabilitation living lab in the mall community of practice: learning together to improve rehabilitation, participation and social inclusion for people living with disabilities. International Journal of Environmental Research and Public Health, 12(4), 4439-4460. https://doi.org/10.3390/ijerph120404439

Meadan, H., Halle, J. W., \& Ebata, A. T. (2010). Families With Children Who Have Autism Spectrum Disorders: Stress and Support [Article]. Exceptional Children, 77(1), 7-36. https://doi.org/10.1177/001440291007700101

Mendez-Ulrich, J. L., Prats-Basset, L., Yague, F., \& Sanz, A. (2016). Perceived control, coping and psychological adjustment to visual disability [Article]. Ansiedad Y Estres-Anxiety and Stress, 22(2-3), 55-61. https://doi.org/10.1016/j.anyes.2016.09.002

Nakade, A., Rohatgi, J., Bhatia, M. S., \& Dhaliwal, U. (2017). Adjustment to acquired vision loss in adults presenting for visual disability certification [Article]. Indian Journal of Ophthalmology, 65(3), 228-232. https://doi.org/10.4103/ijo.IJO_483_16

Oliveira, C. M., \& Nunes, C. H. S. d. S. (2015). Instrumentos para Avaliação Psicológica de Pessoas com Deficiência Visual: Tecnologias para Desenvolvimento e Adaptação Psicologia: Ciência e Profissão, 35, 886-899 https://doi.org/1414-9893

ONU. (2006). Convención de las naciones unidas sobre los derechos de las personas con discapacidad y su protocolo facultativo. Retrieved from http://www.un.org/disabilities/documents/convention/convoptprot-s.pdf

Opoku, M., Alupo, B., Gyamfi, N., Odame, L., Mprah, W., Torgbenu, E., \& Badu, E. (2018). The Family and Disability in Ghana: Highlighting Gaps in Achieving Social Inclusion. Disability, CBR \& Inclusive Development, 28, 41. https://doi.org/10.5463/dcid.v28i4.666

Organización Mundial de la salud, O. (2012). 10 datos sobre la ceguera y la discapacidad visual. http://www.who.int/features/factfiles/blindness/blindness_facts/es/

Peres, R., Espírito-Santo, G., Espírito, F., Ferreira, N., \& Assis, M. (2015). Insatisfação com a imagem corporal entre pessoas com deficiência visual. Revista Brasileira de Ciências do Esporte, 37. https://doi.org/10.1016/j.rbce.2015.08.013

Perez, F. J., Romeo, M., \& Yepes-Baldó, M. (2018). The corporate social responsibility policies for the inclusion of people with disabilities as predictors of employees identification, commitment and absenteeism. Anales De Psicologia, 34, 101-107.

Phillips, B. A., Fortney, S., \& Swafford, L. (2019). College Students' Social Perceptions Toward Individuals With Intellectual Disability [Article]. Journal of Disability Policy Studies, 30(1), 3-10. https://doi.org/10.1177/1044207318788891

Raymond, E., Grenier, A., \& Hanley, J. (2014). Community Participation of Older Adults with Disabilities [Article]. Journal of Community \& Applied Social Psychology, 24(1), 50-62. https://doi.org/10.1002/casp.2173 
Rojas Munera, S., Ruiz Cosme, S., Carvajal Fernández, J., Álvarez, M. J., Duque Restrepo, D., Correa, S. A., Ramírez Rodríguez, C., Pineda Jiménez, L., Restrepo Arismendy, S., Castrillón Valencia, L. C., Arango Valencia, S., Portela, M. C., Fernández Zurita, M., Astudillo Valverde, E., Galarcio, J. F., \& Suárez-Escudero, J. C. (2015). Caracterización de una población con discapacidad visual (baja visión y ceguera) atendida en dos Instituciones Prestadoras de Salud de Medellín. Medicina U.P.B., 34(1), 30-39. https://www.redalyc.org/articulo.oa?id=159046025005 (IN FILE)

Saini, M., \&, \& Shlonsky, A. (2012). Systematic Synthesis of Qualitative Research. In Systematic Synthesis of Qualitative Research. https://oxford.universitypressscholarship.com/view/10.1093/acprof:oso/9780195387216.001.0 001/acprof-9780195387216-chapter-005.

Santos, W. (2016). Deficiência como restrição de participação social: desafios para avaliação a partir da Lei Brasileira de Inclusão. Ciência \& Saúde Coletiva, 21, 3007-3015.

Simplican, S. C., Leader, G., Kosciulek, J., \& Leahy, M. (2015). Defining social inclusion of people with intellectual and developmental disabilities: An ecological model of social networks and community participation [Review]. Research in Developmental Disabilities, 38, 18-29. https://doi.org/10.1016/j.ridd.2014.10.008

Stevens, G. A., White, R. A., Flaxman, S. R., Price, H., Jonas, J. B., Keeffe, J., Leasher, J., Naidoo, K., Pesudovs, K., Resnikoff, S., Taylor, H., \& Bourne, R. R. A. (2013). Global Prevalence of Vision Impairment and Blindness: Magnitude and Temporal Trends, 1990-2010. Ophthalmology, 120(12), 2377-2384. https://doi.org/https://doi.org/10.1016/j.ophtha.2013.05.025

Tobias, E. I., \& Mukhopadhyay, S. (2017). Disability and Social Exclusion: Experiences of Individuals with Visual Impairments in the Oshikoto and Oshana Regions of Namibia [Article]. Psychology and Developing Societies, 29(1), 22-43. https://doi.org/10.1177/0971333616689203

World, Health, \& Organization. (2013). Sixty-sixth World Health Assembly A66/11. Action plan project to prevent avoidable blindness and vision impairment 2014-2019. Retrieved from http://www.emro.who.int/control-and-preventions-of-blindness-anddeafness/announcements/action-plan-prevention-avoidable-blindness-visual-impairment2014-2019.html 